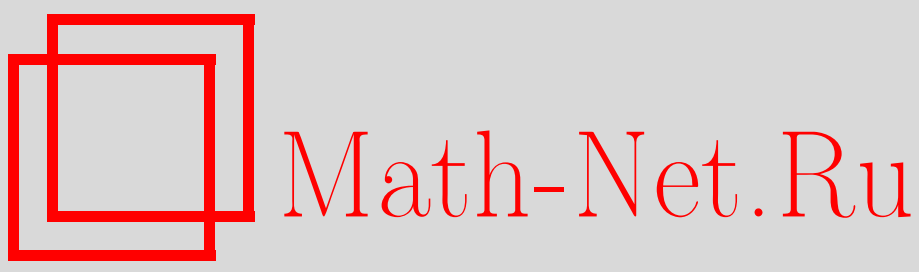

G. D. Hu, A stability criterion for the system of high-order neutral delay differential equations, Sibirsk. Mat. Zh., 2020, Volume 61, Number 6, 1421-1429

DOI: https://doi.org/10.33048/smzh.2020.61.614

Use of the all-Russian mathematical portal Math-Net.Ru implies that you have read and agreed to these terms of use http://www . mathnet.ru/eng/agreement

Download details:

IP : 52.6 .47 .48

April 26, 2023, 16:04:45

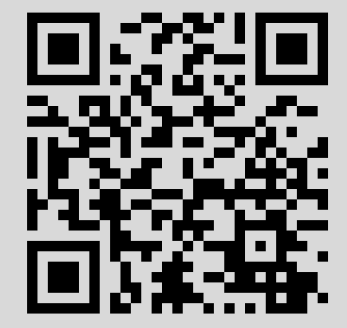


Сибирский математический журнал Ноябрь-декабрь, 2020. Том 61, № 6

УДК 517.925

\title{
КРИТЕРИЙ УСТОЙЧИВОСТИ СИСТЕМЫ ДИФФЕРЕНЦИАЛЬНЫХ УРАВНЕНИЙ НЕЙТРАЛЬНОГО ТИПА ВЫСОКОГО ПОРЯДКА С ЗАПАЗДЫВАНИЕМ
}

\section{Г. Д. Ху}

\begin{abstract}
Аннотация. Изучается устойчивость в зависимости от запаздывания линейных систем нейтрального типа высокого порядка. Сначала выводится оценка для неустойчивых собственных значений для систем нейтрального типа. Оценка неустойчивых собственных значений использует только нормы матриц низшей размерности. Далее с использованием принципа аргумента дается критерий устойчивости, являющийся необходимым и достаточным условием устойчивости в зависимости от запаздывания для систем нейтрального типа. Излагается эффективный численный алгоритм для проверки устойчивости систем нейтрального типа. Обобщаются известные ранее результаты.
\end{abstract}

DOI 10.33048/smzh.2020.61.614

Ключевые слова: устойчивость в зависимости от запаздывания, системы с запаздыванием нейтрального типа высокого порядка, принцип аргумента.

\section{1. Введение}

В статье рассматривается система дифференциальных уравнений с запаздыванием нейтрального типа высокого порядка:

$$
x^{(n)}(t)+\sum_{l=1}^{n}\left[A_{l} x^{(n-l)}(t)+\sum_{j=1}^{m} B_{l j} x^{(n-l)}\left(t-\tau_{j}\right)\right]+\sum_{j=1}^{m} C_{j} x^{(n)}\left(t-\tau_{j}\right)=0
$$

с условием

$$
\sum_{j=1}^{m}\left\|C_{j}\right\|<1
$$

где матрицы параметров $A_{l}, B_{l j}, C_{j} \in \mathbb{R}^{d \times d}, l=1, \ldots, n, j=1, \ldots, m, \tau_{j}>0$, постоянны и индексы при $x$ означают производные по независимой переменной $t$. Всюду в статье предполагается выполнение условия (2). Системы дифференциальных уравнений с запаздыванием высокого порядка возникают в задачах управления, например, в двустороннем управлении системами дистанционного управления [1].

This work is supported by the National Natural Science Foundation of China (11871330).

(c) 2020 Ху Г.Д. 
Устойчивость систем с запаздыванием можно разделить на две категории в зависимости от размера запаздывания. Устойчивость, не зависящая от запаздывания, называется абсолютной устойчивостъю, иначе она называется устойчивостью в зависимости от запаздывания. В данной статье исследуется устойчивость в зависимости от запаздывания систем с запаздыванием нейтрального типа (1) с условием (2).

Если $A_{l}, B_{l j}, C_{j}$ - скаляры, то система (1) сводится к уравнению с запаздыванием нейтрального типа высокого порядка. Устойчивость скалярных уравнений с запаздыванием такого типа изучалась в [2], где дано необходимое и достаточное условие устойчивости в зависимости от запаздывания уравнений с запаздыванием нейтрального типа. Устойчивость одного класса скалярных уравнений с запаздыванием нейтрального типа исследована в [3]. При $n=1$ система (1) сводится к системе первого порядка. Устойчивость систем с запаздыванием нейтрального типа первого порядка исследуется, например, в [2, 4-7]). Настоящая работа является в той или иной степени обобщением ситуации для скалярных уравнений с запаздыванием нейтрального типа и систем с запаздыванием нейтрального типа первого порядка.

Один из методов локализации корней уравнения $g(s)=\operatorname{det} P(s)$ состоит в оценивании характеристической функции $g(s)$, а затем применении к ней признака устойчивости. Скалярный признак устойчивости (см., например, [2]) требует информации о всех коэффициентах характеристической функции $g(s)$. Тем не менее вычисление всех коэффициентов характеристической функции $g(s)$ для больших задач (когда $d$ или $n$, или $m$ велико) некорректно, даже если $g(s)$ - многочлен (см. [8]). Нужно найти прямой признак устойчивости, не использующий вычисления коэффициентов характеристической функции $g(s)$. С другой стороны, в [7] с использованием принципа аргумента получены критерии устойчивости в зависимости от запаздывания для систем нейтрального типа первого порядка. Методы работы [7] избегают вычисления коэффициентов характеристической функции $g(s)$ : за счет введения новых переменных состояния можно переписать систему с запаздыванием нейтрального типа $n$-го порядка (1) с матрицами параметров размера $d \times d$ в виде системы с запаздыванием первого порядка с матрицами параметров размера $n d \times n d$. В теоретическом плане критерии устойчивости из [7] системы с запаздыванием нейтрального типа первого порядка можно применить непосредственно к системе (1). Но это сопряжено с большими вычислительными проблемами.

В случае $C_{j}=0$ для $j=1, \ldots, m$ система (1) сводится к следующей системе с запаздывание $n$-го порядка:

$$
x^{(n)}(t)+\sum_{l=1}^{n}\left[A_{l} x^{(n-l)}(t)+\sum_{j=1}^{m} B_{l j} x^{(n-l)}\left(t-\tau_{j}\right)\right]=0 .
$$

Недавно в [6] получены критерии устойчивости в зависимости от запаздывания для системы (3). Критерии в [6] не используют вычисление коэффициентов характеристической матрицы, а используют только нормы $d \times d$-матриц, которые могут хорошо работать на практике для больших задач (когда $d$ или $n$, или $m$ велико). В настоящей работе продолжаются исследования, начатые в $[6,7]$, и обобщаются содержащиеся там результаты на системы с запаздыванием нейтрального типа высокого порядка (1) с условием (2). Насколько известно автору, в литературе нет результатов об устойчивости системы с запаздыванием (1) нейтрального типа с условием (2). 
Основные результаты настоящей работы можно сформулировать следующим образом.

1. Предлагается необходимое и достаточное условие устойчивости в зависимости от запаздывания линейных систем дифференциальных уравнений с запаздыванием нейтрального типа высокого порядка.

2. На основе необходимого и достаточного условия предложен эффективный численный алгоритм проверки устойчивости в зависимости от запаздывания нейтральных систем.

Всюду далее $j$-е собственное значение $A$ обозначается символом $\lambda_{j}(A), \rho(A)$ представляет собой спектральный радиус и $\|A\|$ означает матричную норму $A$. Открытая левая полуплоскость $\{s: \operatorname{Re} s<0\}$ обозначается символом $\mathbb{C}^{-}$, а правая полуплоскость $\{s: \operatorname{Re} s \geq 0\}-$ символом $\mathbb{C}^{+}$.

\section{2. Предварительные сведения}

Характеристическая функция системы (1) имеет следующий вид:

$$
g(s)=\operatorname{det} P(s),
$$

где $P(s)$ определяется так:

$$
P(s)=I s^{n}+\sum_{l=1}^{n} A_{l} s^{n-l}+\sum_{l=1}^{n} \sum_{j=1}^{m} B_{l, j} s^{n-l} \exp \left(-\tau_{j} s\right)+\sum_{j=1}^{m} C_{j} s^{n} \exp \left(-\tau_{j} s\right) .
$$

Если $g(\xi)=0$, то $\xi$ называется собственным значением системы (1). Если $\operatorname{Re} \xi<0$, то собственное значение $\xi$ называется устойчивым, иначе - неустойчивым.

Перепишем систему (1) в виде системы с запаздыванием нейтрального типа первого порядка. Пусть

$$
\begin{aligned}
y_{1}(t) & =x(t), \\
y_{2}(t) & =x^{(1)}(t)=\dot{y}_{1}(t), \\
& \vdots \\
y_{n}(t) & =x^{(n-1)}(t)=\dot{y}_{n-1}(t) .
\end{aligned}
$$

Из (1) имеем

$$
\begin{aligned}
\dot{y}_{n}(t)=x^{(n)}(t)=-\left\{\sum_{l=1}^{n}\left[A_{l} y_{n-l+1}(t)+\sum_{j=1}^{m} B_{l j} y_{n-l+1}\left(t-\tau_{j}\right)\right]\right. & \\
& \left.+\sum_{j=1}^{m} C_{j} \dot{y}_{n}\left(t-\tau_{j}\right)\right\},
\end{aligned}
$$

что приводит к системе дифференциальных уравнений нейтрального типа первого порядка [9]:

$$
\dot{Y}(t)=\mathscr{A} Y(t)+\sum_{j=1}^{m} \mathscr{B}_{j} Y\left(t-\tau_{j}\right)+\sum_{j=1}^{m} \mathscr{C}_{j} \dot{Y}\left(t-\tau_{j}\right)
$$


где $Y(t)=\left[y_{1}(t), y_{2}(t), \ldots, y_{n}(t)\right]^{T}$,

$$
\begin{gathered}
\mathscr{A}=\left[\begin{array}{ccccc}
0 & I & 0 & \ldots & 0 \\
0 & 0 & I & & \vdots \\
\vdots & \vdots & \vdots & & I \\
-A_{n} & -A_{n-1} & -A_{n-2} & \ldots & -A_{1}
\end{array}\right], \\
\mathscr{B}_{j}=\left[\begin{array}{ccccc}
0 & 0 & 0 & \ldots & 0 \\
\vdots & \vdots & \vdots & & \vdots \\
0 & 0 & 0 & \ldots & 0 \\
-B_{n, j} & -B_{n-1, j} & -B_{n-2, j} & \ldots & -B_{1, j}
\end{array}\right], \\
\mathscr{C}_{j}=\left[\begin{array}{ccccc}
0 & 0 & 0 & \ldots & 0 \\
\vdots & \vdots & \vdots & & \vdots \\
0 & 0 & 0 & \ldots & 0 \\
0 & 0 & 0 & \ldots & -C_{j}
\end{array}\right]
\end{gathered}
$$

Заметим, что

$$
\begin{aligned}
\mathscr{A}+\sum_{j=1}^{m} \mathscr{B}_{j} \exp \left(-\tau_{j} s\right) & +\sum_{j=1}^{m} \mathscr{C}_{j} \exp \left(-\tau_{j} s\right) s \\
& =\left[\begin{array}{ccccc}
0 & I & 0 & \ldots & 0 \\
0 & 0 & I & & \vdots \\
\vdots & \vdots & \vdots & & I \\
-\widehat{A}_{n}(s) & -\widehat{A}_{n-1}(s) & -\widehat{A}_{n-2}(s) & \ldots & -\widehat{A}_{1}(s)
\end{array}\right]
\end{aligned}
$$

где

$$
\begin{gathered}
\widehat{A}_{1}(s)=A_{1}+\sum_{j=1}^{m} B_{1, j} \exp \left(-\tau_{j} s\right)+\sum_{j=1}^{m} C_{j} s \exp \left(-\tau_{j} s\right), \widehat{A}_{2}(s)=A_{2}+\sum_{j=1}^{m} B_{2, j} \exp \left(-\tau_{j} s\right), \\
\ldots, \widehat{A}_{n-2}(s)=A_{n-2}+\sum_{j=1}^{m} B_{n-2, j} \exp \left(-\tau_{j} s\right), \\
\widehat{A}_{n-1}(s)=A_{n-1}+\sum_{j=1}^{m} B_{n-1, j} \exp \left(-\tau_{j} s\right), \quad \widehat{A}_{n}(s)=A_{n}+\sum_{j=1}^{m} B_{n, j} \exp \left(-\tau_{j} s\right) .
\end{gathered}
$$

В силу линеаризации матричного полинома [9] и (5)

$$
\begin{aligned}
& \operatorname{det}\left\{s I-\left[\mathscr{A}+\sum_{j=1}^{m} \mathscr{B}_{j} \exp \left(-\tau_{j} s\right)+\sum_{j=1}^{m} \mathscr{C}_{j} s \exp \left(-\tau_{j} s\right)\right]\right\} \\
& =\operatorname{det}\left\{I s^{n}+\widehat{A}_{1}(s) s^{n-1}+\widehat{A}_{2}(s) s^{n-2}+\cdots+\widehat{A}_{n}(s)\right\} \\
& =\operatorname{det}\left\{I s^{n}+A_{1} s^{n-1}+\sum_{j=1}^{m} B_{1, j} \exp \left(-\tau_{j} s\right) s^{n-1}+\sum_{j=1}^{m} C_{j} s^{n} \exp \left(-\tau_{j} s\right)\right. \\
& \left.+A_{2} s^{n-2}+\sum_{j=1}^{m} B_{2, j} \exp \left(-\tau_{j} s\right) s^{n-2}+\cdots+A_{n}+\sum_{j=1}^{m} B_{n, j} \exp \left(-\tau_{j} s\right)\right\} \\
& =\operatorname{det}\left\{I s^{n}+\sum_{l=1}^{n} A_{l} s^{n-l}+\sum_{l=1}^{n} \sum_{j=1}^{m} B_{l, j} s^{n-l} \exp \left(-\tau_{j} s\right)+\sum_{j=1}^{m} C_{j} s^{n} \exp \left(-\tau_{j} s\right)\right\}=\operatorname{det} P(s),
\end{aligned}
$$


T. e.

$$
\operatorname{det}\left\{s I-\left[\mathscr{A}+\sum_{j=1}^{m} \mathscr{B}_{j} \exp \left(-\tau_{j} s\right)+\sum_{j=1}^{m} \mathscr{C}_{j} s \exp \left(-\tau_{j} s\right)\right]\right\}=\operatorname{det} P(s) .
$$

Дадим следующее определение устойчивости системы (1).

ОПРедЕЛЕНИЕ 2.1. Система с запаздыванием (1) нейтрального типа высокого порядка называется асимптотически устойчивой, если система с запаздыванием нейтрального типа первого порядка (6) асимптотически устойчива.

Лемма 2.1. Система (1) с условием (2) асимптотически устойчива тогда и только тогда, когда все ее собственные значения лежат в открытой левой комплексной полуплоскости.

ДокАЗАТЕЛЬСтво. Согласно определению 2.1 система (1) асимптотически устойчива в том и только в том случае, если система (6) асимптотически устойчива. В силу определения $\mathscr{C}_{j}$ получаем, что $\omega_{j} \in[0,2 \pi], j=1,2, \ldots, m$, и

$$
\rho\left(\sum_{j=1}^{m} \mathscr{C}_{j} \exp \left(i \omega_{j}\right)\right)=\rho\left(\sum_{j=1}^{m}-C_{j} \exp \left(i \omega_{j}\right)\right) \leq \sum_{j=1}^{m}\left\|C_{j}\right\|<1,
$$

где $i^{2}=-1$. Значит, разностный оператор в системе (6) сильно устойчив при условии (2) [5]. Система (6) с условием (2) асимптотически устойчива тогда и только тогда, когда все корни характеристической функции

$$
\operatorname{det}\left\{s I-\left[\mathscr{A}+\sum_{j=1}^{m} \mathscr{B}_{j} \exp \left(-\tau_{j} s\right)+\sum_{j=1}^{m} \mathscr{C}_{j} s \exp \left(-\tau_{j} s\right)\right]\right\}=0
$$

лежат в открытой левой полуплоскости (см., например, [5]). В силу (10) это означает, что все корни уравнения $g(s)=0(g(s)=\operatorname{det} P(s))$ лежат в открытой левой комплексной полуплоскости. Лемма доказана.

ЗАмечАние 2.1. Теоретически анализ устойчивости системы (1) с условием (2) можно свести к анализу системы (6) с условием (2). Таким образом, можно прямо применить критерий устойчивости из [7]. Однако поскольку $\mathscr{A}, \mathscr{B}_{j}$ и $\mathscr{C}_{j}$ принадлежат $\mathbb{R}^{n d \times n d}$ для $j=1,2, \ldots, m$, требуется много вычислительных усилий для больших задач ввиду того, что матрицы имеют размер $n d \times n d$.

\section{3. Оценка неустойчивых собственных значений}

В этом разделе выводится оценка для неустойчивых собственных значений системы (1) с условием (2). Оценка использует только нормы матриц размера $d \times d$.

Теорема 3.1. Всякое неустойчивое собственное значение $\xi$ системы (1) с условием (2) удовлетворяет неравенству

$$
|\xi| \leq \beta=\max \{1, a\}
$$

где скаляр а определяется равенством

$$
a=\frac{\sum_{l=1}^{n}\left\|A_{l}\right\|+\sum_{l=1}^{n} \sum_{j=1}^{m}\left\|B_{l j}\right\|}{1-\sum_{j=1}^{m}\left\|C_{j}\right\|} .
$$


ДокАЗАтЕльство. Пусть $\xi-$ собственное значение системы (1), т. е. $g(\xi)=0$. Так как $\xi-$ неустойчивый корень, $\operatorname{Re} \xi \geq 0$. Сначала рассмотрим случай $|\xi| \geq 1$. Согласно (4) имеем

$$
g(\xi)=\operatorname{det} P(\xi)=0,
$$

откуда следует, что

$$
\operatorname{det}\left[I \xi^{n}+\sum_{l=1}^{n} A_{l} \xi^{n-l}+\sum_{l=1}^{n} \sum_{j=1}^{m} B_{l j} \xi^{n-l} \exp \left(-\tau_{j} \xi\right)+\sum_{j=1}^{m} C_{j} \xi^{n} \exp \left(-\tau_{j} \xi\right)\right]=0 .
$$

Так как $\operatorname{Re} \xi \geq 0$, то

$$
\left|\exp \left(-\tau_{j} \xi\right)\right| \leq 1
$$

Из условия (2) и неравенства (15) получаем

$$
\left\|\sum_{j=1}^{m} C_{j} \exp \left(-\tau_{j} \xi\right)\right\| \leq \sum_{j=1}^{m}\left\|C_{j}\right\|<1 .
$$

Известно [9], что $\left(I-\sum_{j=1}^{m} C_{j} \exp \left(-\tau_{j} \xi\right)\right)^{-1}$ существует и

$$
\left\|I-\sum_{j=1}^{m} C_{j} \exp \left(-\tau_{j} \xi\right)\right\|^{-1} \leq \frac{1}{1-\left\|\sum_{j=1}^{m} C_{j} \exp \left(-\tau_{j} \xi\right)\right\|} \leq \frac{1}{1-\sum_{j=1}^{m}\left\|C_{j}\right\|} .
$$

Для $|\xi| \geq 1$ и $\operatorname{Re} \xi \geq 0$ введем матрицу

$$
W(\xi)=-\left(I-\sum_{j=1}^{m} C_{j} \exp \left(-\tau_{j} \xi\right)\right)^{-1}\left(\sum_{l=1}^{n} A_{l} \xi^{1-l}+\sum_{l=1}^{n} \sum_{j=1}^{m} B_{l j} \xi^{1-l} \exp \left(-\tau_{j} \xi\right)\right) .
$$

Так как $|\xi| \geq 1$, перепишем (14) в виде

$$
\operatorname{det} P(\xi)=\operatorname{det}\left[\xi^{n-1}\left(I-\sum_{j=1}^{m} C_{j} \exp \left(-\tau_{j} \xi\right)\right)\right] \operatorname{det}[I \xi-W(\xi)]=0 .
$$

Отсюда

$$
\operatorname{det}[\xi I-W(\xi)]=0
$$

Следовательно, $\xi$ - собственное значение матрицы $W(\xi)$, и найдется целое число $j(1 \leq j \leq d)$ такое, что

$$
\xi=\lambda_{j}(W(\xi))
$$

Поскольку $|\xi| \geq 1$, для $k \geq 0$ будет

$$
\left|\xi^{-k}\right| \leq 1
$$

Используя (20), имеем [9]

$$
|\xi|=\left|\lambda_{j}(W(\xi))\right| \leq\|W(\xi)\| .
$$

Ввиду (18), определения $W(s),(15),(17)$ и $(21)$

$$
\|W(\xi)\|=\left\|\left(I-\sum_{j=1}^{m} C_{j} \exp \left(-\tau_{j} \xi\right)\right)^{-1}\left(\sum_{l=1}^{n} A_{l} \xi^{1-l}+\sum_{l=1}^{n} \sum_{j=1}^{m} B_{l j} \xi^{1-l} \exp \left(-\tau_{j} \xi\right)\right)\right\|
$$


Критерий устойчивости системы дифференциальных уравнений 1427

$$
\begin{gathered}
\leq\left\|\left(I-\sum_{j=1}^{m} C_{j} \exp \left(-\tau_{j} \xi\right)\right)^{-1}\right\|\left\|\sum_{l=1}^{n} A_{l} \xi^{1-l}+\sum_{l=1}^{n} \sum_{j=1}^{m} B_{l j} \xi^{1-l} \exp \left(-\tau_{j} \xi\right)\right\| \\
\leq \frac{1}{1-\sum_{j=1}^{m}\left\|C_{j}\right\|}\left(\sum_{l=1}^{n}\left\|A_{l} \xi^{1-l}\right\|+\sum_{l=1}^{n} \sum_{j=1}^{m}\left\|B_{l j} \xi^{1-l} \exp \left(-\tau_{j} \xi\right)\right\|\right) \\
\leq \frac{\sum_{l=1}^{n}\left\|A_{l}\right\|+\sum_{l=1}^{n} \sum_{j=1}^{m}\left\|B_{l j}\right\|}{1-\sum_{j=1}^{m}\left\|C_{j}\right\|}=a .
\end{gathered}
$$

Используя (22), получаем, что для $|\xi| \geq 1$ и $\operatorname{Re} \xi \geq 0$ выполнено $|\xi| \leq a$. Для любого собственного значения $\xi$ со свойством $\operatorname{Re} \xi \geq 0$ имеем

$$
|\xi| \leq \beta=\max \{1, a\} .
$$

Теорема доказана.

\section{4. Численный алгоритм для анализа устойчивости}

Построим численный алгоритм исследования устойчивости в зависимости от запаздывания для системы (1) с условием (2).

Проверим существование собственных значений у системы (1) с условием (2), лежащих в правой комплексной полуплоскости. Для системы (1) с условием (2) определим область $D=\{s: \operatorname{Re} s \geq 0,|s| \leq \beta\}$, границу которой обозначим через $C$. Здесь $\beta$ такое же, как в формуле (12).

Из определения области $D$ очевидно, что $D \subset \mathbb{C}^{+}$. Следующая теорема исключит все неустойчивые собственные значения системы (1) с условием (2) из области $D$. Необходимое и достаточное условие для устойчивости в зависимости от запаздывания системы (1) с условием (2) выводится с помощью принципа аргумента.

Теорема 4.1. Система (1) с условием (2) асимптотически устойчива тогда и только тогда, когда

$$
g(s) \neq 0 \quad \text { для } s \in C
$$

и

$$
\Delta_{C} \arg g(s)=0 .
$$

Здесь $g(s)=\operatorname{det} P(s), \arg g(s)-$ аргумент $g(s)$ и $\Delta_{C} \arg g(s)$ - изменение аргумента $g(s)$ вдоль кривой $C$.

ДокАЗАТЕЛЬСтво. Предположим, что система (1) с условием (2) асимптотически устойчива. По лемме 2.1 все нули $g(s)$ расположены в левой полуплоскости $\mathbb{C}^{-}$. Значит, $g(s) \neq 0$ при $\operatorname{Re} s \geq 0$. Согласно принципу аргумента [10] приходим к соотношениям (23) и (24).

Обратно, предположим, что условия (23) и (24) выполнены. Согласно теореме 3.1 если существуют неустойчивые собственные значения характеристической функции $g(s)$, то неустойчивые собственные значения должны лежать в области $D$. Используя принцип аргумента [10] и условия (23) и (24), получаем, что $g(s)$ не обращается в нуль для $\operatorname{Re} s \geq 0$. Поэтому из $(23)$ и (24) следует, что система (1) с условием (2) асимптотически устойчива. Теорема доказана. 
Опишем алгоритм проверки устойчивости в зависимости от запаздывания системы (1) с условием (2) в силу теоремы 4.1 .

ШАГ 0. Вычислить $\beta$ по (12). Тогда границей области $D$ является замкнутый контур $C$, который состоит из двух частей, а именно из сегмента $\{s=i t ;-\beta \leq t \leq \beta\}$ и полукруга $\{s ;|s|=\beta,-\pi / 2 \leq \arg s \leq \pi / 2\}$. Заметим, что контур $C$ положительно ориентирован, когда он проходится против часовой стрелки.

ШАГ 1. Возьмем достаточно большое целое число $N \in \mathbb{N}$ и распределим $N$ узловых точек $\left\{s_{j}\right\}, j=1,2, \ldots, N$, на $C$ насколько возможно равномерно. Для каждого $s_{j}$ оценим $g\left(s_{j}\right)$, вычисляя определитель следующим образом:

$$
g\left(s_{j}\right)=\operatorname{det} P\left(s_{j}\right) .
$$

Разложим также $g\left(s_{j}\right)$ на вещественную и мнимую части для вычисления аргумента.

ШАГ 2. Исследуем, верно ли, что $g\left(s_{j}\right)=0$ для каждого $s_{j}, j=1, \ldots, N$, проверив, удовлетворяет ли его модуль неравенству $\left|g\left(s_{j}\right)\right| \leq \delta_{1}$ с любым наперед заданным $\delta_{1}$. Если это верно, т. е. $s_{j} \in C-$ корень $g(s)$, то система (1) с условием (2) неустойчива и алгоритм останавливается. Иначе перейти на следующий шаг.

ШАГ 3. Исследуем, верно ли, что $\Delta_{C} \arg g(s)=0$ вдоль последовательности $\left\{g\left(s_{j}\right)\right\}$, проверив, что $\left|\Delta_{C} \arg g(s)\right| \leq \delta_{2}$ с наперед заданной точностью $\delta_{2}$. Если это верно, то изменение аргумента равно нулю вдоль $C$. Тогда система (1) с условием (2) асимптотически устойчива; иначе - неустойчива.

В изложенном алгоритме отсутствуют вычисления коэффициентов характеристической функции $g(s)=\operatorname{det} P(s)$. Вместо этого есть оценка определителя числовой матрицы $P\left(s_{j}\right)$ посредством элементарных операций со строками (или столбцами), которые относительно эффективны [8]. Скалярный критерий устойчивости [2] требует информации о всех коэффициентах характеристической функции $g(s)$, вычисление которых для больших задач некорректно, даже если $g(s)$ - многочлен [8]. Хотя теоретически можно получить коэффициенты характеристической функции $g(s)$ из $P(s)$, возможно, это плохо сработает для больших задач на практике.

Исследуем число неустойчивых собственных значений системы (1) с условием (2).

Теорема 4.2. Если

$$
g(s) \neq 0 \quad \text { для } s \in C
$$

и

$$
\frac{1}{2 \pi} \Delta_{C} \arg g(s)=z
$$

то число неустойчивых собственных значений системы (1) с условием (2) равно $z$. Здесь $g(s)=\operatorname{det} P(s)$

ДокАЗАТЕЛЬство. В силу теоремы 3.1 все неустойчивые характеристические корни расположены в области $D$. С использованием условия $(25)$ и принципа аргумента [10] число неустойчивых характеристических корней вычисляется по формуле (26). Теорема доказана.

ЗАмечАниЕ 4.1. Если на границе $C$ имеется $z_{0}$ собственных значений, то можно построить модифицированную кривую [11], заменяющую границу $C$. 
Теорему 4.2 можно обобщить, и число устойчивых собственных значений равно $z_{0}+z$.

ЗАмечАние 4.2. Модифицируя лишь шаг 3 алгоритма 1, т. е. вычисляя $\Delta_{C} \arg g(s)$ вдоль последовательности $\left\{g\left(s_{j}\right)\right\}$, можно проверить теорему 4.2.

ЗАмечАниЕ 4.3. В [6] представлена процедура, аналогичная алгоритму 1 , для проверки устойчивости системы дифференциальных уравнений с запаздыванием высокого порядка (3).

\section{ЛИТЕРАТУРА}

1. Islam S., Liu P. X., Saddik A. E., Yang Y. B. Bilateral control of teleoperation systems with time delay // IEEE/ASME Trans. Mechatroics. 2015. V. 20. P. 1-12.

2. Kolmanovskii V. B., Myshkis A. Introduction to theory and applications of functional differential equations. Dordrecht: Kluwer Acad. Publ., 1999.

3. Cahlon B., Schmidt D. Stability criteria for certain high odd order delay differential equations // J. Comput. Appl. Math. 2007. V. 200. P. 408-423.

4. Demidenko G. V., Matveeva I. I. On exponential stability of solutions to one class of systems of differential equations of neutral type // J. Appl. Indust. Math. 2014. V. 8. P. 510-520.

5. Hale J. K., Verduyn Lunel S. M. Strong stabilization of neutral functional differential equations // IMA J. Math. Control Info. 2002. V. 19. P. 5-23.

6. $\mathrm{Hu}$ G. D. Stability criteria of high order delay differential systems. Int. J. Contr. 2018. doi:10.1080/00207179.2018.1541365.

7. Hu G. D., Liu M. Stability criteria of linear neutral systems with multiple delays // IEEE Trans. Autom. Contr. 2007. V. 52. P. 720-724.

8. Johnson L. W., Dean-Riess R., Arnold J. T. Introduction to linear algebra. Prentice-Hall: Englewood Cliffs, 2000.

9. Lancaster P., Tismenetsky $M$. The theory of matrices with applications. Orlando: Acad. Press, 1985.

10. Brown J. W., Churchill R. V. Complex variables and applications. Beijing: McGraw-Hill Comp., Inc.; China Machine Press, 2004.

11. Franklin G. F., Powell J. D., Emami-Naeini A. Feedback control of dynamic systems. New York: Addison-Wesley Publ. Comp., 1994.

Поступила в редакцию 30 декабря 2019 г.

После доработки 30 декабря 2019 г.

Принята к публикации 19 февраля 2020 г.

Guan-Da Hu (Ху Гуан-Да)

Department of Mathematics

Shanghai University, Shanghai, 200444, China

ghu@hit.edu.cn 